\title{
REVERSE ORDER LAW FOR THE MOORE-PENROSE INVERSE IN $C^{*}$-ALGEBRAS*
}

\author{
DIJANA MOSIĆ ${ }^{\dagger}$ AND DRAGAN S. DJORDJEVIĆ ${ }^{\dagger}$
}

\begin{abstract}
In this paper, several equivalent conditions related to the reverse order law for the Moore-Penrose inverse in $C^{*}$-algebras are studied. Some well-known results are extended to more general settings. Then this result is applied to obtain the reverse order rule for the weighted Moore-Penrose inverse in $C^{*}$-algebras.
\end{abstract}

Key words. Moore-Penrose inverse, Reverse order law, $C^{*}$-algebras.

AMS subject classifications. 16W10, 15A09, 46L05.

1. Introduction. Let $S$ be a semigroup with the unit 1 . If $a, b \in S$ are invertible, then $a b$ is invertible too and the inverse of the product $a b$ satisfied the reverse order law $(a b)^{-1}=b^{-1} a^{-1}$. This formula cannot trivially be extended to the MoorePenrose inverse of the product $a b$. In this paper, we investigate necessary and sufficient conditions which are related to the reverse order law for the Moore-Penrose inverse. The reverse order law for the weighted Moore-Penrose inverse in $C^{*}$-algebras follows as a corollary.

Let $\mathcal{A}$ be a unital $C^{*}$-algebra. An element $a \in \mathcal{A}$ is regular if there exists some $b \in \mathcal{A}$ satisfying $a b a=a$. The set of all regular elements of $\mathcal{A}$ will be denoted by $\mathcal{A}^{-}$. An element $p \in \mathcal{A}$ is idempotent if $p^{2}=p$. An element $a \in \mathcal{A}$ is self-adjoint if $a^{*}=a$. An element $x \in \mathcal{A}$ is positive if $x=x^{*}$ and $\sigma(x) \subseteq[0,+\infty)$, where the spectrum of element $x$ is denoted by $\sigma(x)$. Notice that, positive elements are self-adjoint. If $x \in \mathcal{A}$, then $x^{*} x$ is positive element.

The Moore-Penrose inverse (or MP-inverse) of $a \in \mathcal{A}$ is the element $b \in \mathcal{A}$, if the following equations hold [13]:

$$
\text { (1) } a b a=a, \quad(2) b a b=b, \quad(3)(a b)^{*}=a b, \quad \text { (4) }(b a)^{*}=b a .
$$

There is at most one $b$ such that above conditions hold (see [13]), and such $b$ is denoted by $a^{\dagger}$. The set of all Moore-Penrose invertible elements of $\mathcal{A}$ will be denoted by $\mathcal{A}^{\dagger}$.

\footnotetext{
${ }^{*}$ Received by the editors on July 24, 2010. Accepted for publication on January 31, 2011. Handling Editor: Michael Tsatsomeros.

${ }^{\dagger}$ Faculty of Sciences and Mathematics, University of Niš, P.O. Box 224, 18000 Niš, Serbia (dijana@pmf.ni.ac.rs, dragan@pmf.ni.ac.rs). The authors were supported by the Ministry of Science, Republic of Serbia, grant no. 174007.
} 
If $a$ is invertible, then $a^{\dagger}$ coincides with the ordinary inverse of $a$.

Definition 1.1. Let $\mathcal{A}$ be a unital $C^{*}$-algebra and $e, f$ two invertible positive elements in $\mathcal{A}$. We say that the element $a \in \mathcal{A}$ has the weighted MP-inverse with weights $e, f$ if there exists $b \in \mathcal{A}$ such that
(1) $a b a=a$,
(2) $b a b=b$,
$(3 e)(e b a)^{*}=e b a$
$(4 f)(f a b)^{*}=f a b$.

The unique weighted MP-inverse with weights $e, f$, will be denoted by $a_{e, f}^{\dagger}$ if it exists [11]. The set of all weighted MP-invertible elements of $\mathcal{A}$ with weights $e, f$, will be denoted by $\mathcal{A}_{e, f}^{\dagger}$.

If $\delta \subset\{1,2,3,4,3 e, 4 f\}$ and $b$ satisfies the equations $(i)$ for all $i \in \delta$, then $b$ is an $\delta$-inverse of $a$. The set of all $\delta$-inverse of $a$ is denote by $a\{\delta\}$. If $b \in a\{1\}$, then $a$ is regular. If $b \in a\{1,2\}$ and $a b=b a$, then $a$ is group invertible.

Let $\mathcal{R}$ be a ring with involution. An element $a \in \mathcal{R}$ is: left ${ }^{*}$-cancellable if $a^{*} a x=a^{*} a y$ implies $a x=a y$; it is right ${ }^{*}$-cancellable if $x a a^{*}=y a a^{*}$ implies $x a=y a$; and it is *-cancellable if it is both left and right $*^{*}$-cancellable. We observe that $a$ is

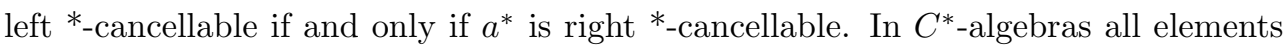
are ${ }^{*}$-cancellable. A ring $\mathcal{R}$ is called ${ }^{*}$-reducing if every element of $\mathcal{R}$ is ${ }^{*}$-cancellable. This is equivalent to the implication $a^{*} a=0 \Rightarrow a=0$ for all $a \in \mathcal{R}$.

Now we formulate the following result, which is well-known and frequently used in the rest of the paper.

THEOREM 1.2. [6, 12] For any $a \in \mathcal{A}^{\dagger}$, the following are satisfied:
(a) $\left(a^{\dagger}\right)^{\dagger}=a$;
(b) $\left(a^{*}\right)^{\dagger}=\left(a^{\dagger}\right)^{*}$;
(c) $\left(a^{*} a\right)^{\dagger}=a^{\dagger}\left(a^{\dagger}\right)^{*}$;
(d) $\left(a a^{*}\right)^{\dagger}=\left(a^{\dagger}\right)^{*} a^{\dagger}$;
(f) $a^{*}=a^{\dagger} a a^{*}=a^{*} a a^{\dagger}$;
(g) $a^{\dagger}=\left(a^{*} a\right)^{\dagger} a^{*}=a^{*}\left(a a^{*}\right)^{\dagger}$;
(h) $\left(a^{*}\right)^{\dagger}=a\left(a^{*} a\right)^{\dagger}=\left(a a^{*}\right)^{\dagger} a$.

TheOREM 1.3. [8] In a unital $C^{*}$-algebra $\mathcal{A}, a \in \mathcal{A}$ is $M P$-invertible if and only if a is regular.

It is useful to express the weighted MP-inverse in terms of the ordinary MPinverse.

TheOREM 1.4. [11] Let $\mathcal{A}$ be a unital $C^{*}$-algebra and let e, $f$ be positive invertible elements of $\mathcal{A}$. If $a \in \mathcal{A}$ is regular, then the unique weighted MP-inverse $a_{e, f}^{\dagger}$ exists and

$$
a_{e, f}^{\dagger}=e^{-1 / 2}\left(f^{1 / 2} a e^{-1 / 2}\right)^{\dagger} f^{1 / 2}
$$


In [7], Greville proved that $(a b)^{\dagger}=b^{\dagger} a^{\dagger}$ holds for complex matrices if and only if $a^{\dagger} a$ commutes with $b b^{*}$ and $b b^{\dagger}$ commutes with $a a^{*}$. Bouldin [2, 3] and Izumino [10] generalized this result for closed range operators on Hilbert spaces. Their proofs are based on operator theoretical methods and use properties of ranges of operators and gaps between subspaces. In [11], a proof of the reverse order rule for the MoorePenrose inverse in the setting of rings with involution is presented, extending the results for Hilbert space operators from $[2,3,10]$. This result is formulated as follows.

TheOREM 1.5. [11] Let $\mathcal{R}$ be a ring with involution, let $a, b \in \mathcal{R}$ be $M P$-invertible and let $\left(1-a^{\dagger} a\right) b$ be left ${ }^{*}$-cancellable. Then the following conditions are equivalent:

(a) $a b$ is MP-invertible and $(a b)^{\dagger}=b^{\dagger} a^{\dagger}$;

(b) $a^{\dagger} a b b^{*}=b b^{*} a^{\dagger} a$ and $b b^{\dagger} a^{*} a=a^{*} a b b^{\dagger}$.

Necessary and sufficient conditions for the reverse order rule for the weighted MP-inverse for matrices were given by Sun and Wei in [14] in terms of the inclusion of matrix ranges (column spaces). In [11], the result for the reverse order rule for the weighted Moore-Penrose inverse in $C^{*}$-algebras is proved, generalizing the matrix results in [14].

Tian $[15,16]$ studied a group of rank equalities related to the Moore-Penrose inverse of products of two matrices, which implies necessary and sufficient conditions for $(a b)^{\dagger}=b^{\dagger} a^{\dagger}$. The extensions of these results to the weighted Moore-Penrose inverse are considered too. The operator analogues of these results for the MoorePenrose inverse are proved in [4, 5] for linear bounded operators on Hilbert spaces, using the matrix form of operators induced by some natural decomposition of Hilbert spaces.

In this paper, we present a purely algebraic proof of some equivalent conditions related to the reverse order law for the Moore-Penrose inverse in $C^{*}$-algebras, extending the known results for matrices $[15,16]$ and Hilbert space operators $[4,5]$. We show that neither the rank (in the finite dimensional case) nor the properties of operator matrices (in the infinite dimensional case) are necessary for the proof of the reverse order rule for the Moore-Penrose inverse valid under certain conditions on regular elements. Thus, we extend some recent results to more general settings. As a corollary, we obtain the reverse order law for the weighted Moore-Penrose inverse.

2. Reverse order law in $C^{*}$-algebras. In this section, we present necessary and sufficient conditions for the reverse order law for the Moore-Penrose inverse to hold. The first list of some equivalent statements is given below. 
Theorem 2.1. Let $\mathcal{A}$ be a unital $C^{*}$-algebra and let $a, b \in \mathcal{A}^{-}$. Then the following conditions are equivalent:
(a) $a b b^{\dagger} a^{\dagger} a b=a b$;
(b) $b^{\dagger} a^{\dagger} a b b^{\dagger} a^{\dagger}=b^{\dagger} a^{\dagger}$;
(c) $a^{\dagger} a b b^{\dagger}=b b^{\dagger} a^{\dagger} a$;
(d) $a^{\dagger} a b b^{\dagger}$ is an idempotent;
(e) $b b^{\dagger} a^{\dagger} a$ is an idempotent;
(f) $b^{\dagger}\left(a^{\dagger} a b b^{\dagger}\right)^{\dagger} a^{\dagger}=b^{\dagger} a^{\dagger}$;
(g) $\left(a^{\dagger} a b b^{\dagger}\right)^{\dagger}=b b^{\dagger} a^{\dagger} a$.

Proof. We can easy get that $a b \in \mathcal{A}^{-} \Leftrightarrow a^{\dagger} a b b^{\dagger} \in A^{-}$.

(a) $\Rightarrow$ (c): From $a b b^{\dagger} a^{\dagger} a b=a b$, we obtain $a^{\dagger} a b b^{\dagger}\left(1-a^{\dagger} a\right) b=0$ and

$$
\begin{aligned}
a^{\dagger} a\left(b^{\dagger}\right)^{*}\left(\left(1-a^{\dagger} a\right) b\right)^{*}\left(1-a^{\dagger} a\right) b & =a^{\dagger} a\left(b^{\dagger}\right)^{*} b^{*}\left(1-a^{\dagger} a\right)\left(1-a^{\dagger} a\right) b \\
& =a^{\dagger} a b b^{\dagger}\left(1-a^{\dagger} a\right) b \\
& =0 .
\end{aligned}
$$

Because all elements are ${ }^{*}$-cancellable in $C^{*}$-algebra, we get

$$
a^{\dagger} a\left(b^{\dagger}\right)^{*}\left(\left(1-a^{\dagger} a\right) b\right)^{*}=0
$$

This equality implies $a^{\dagger} a b b^{\dagger}\left(1-a^{\dagger} a\right)=0$, i.e.,

$$
a^{\dagger} a b b^{\dagger}=a^{\dagger} a b b^{\dagger} a^{\dagger} a .
$$

Now, by (2.2), we have

$$
a^{\dagger} a b b^{\dagger}=a^{\dagger} a b b^{\dagger} a^{\dagger} a=\left(a^{\dagger} a b b^{\dagger} a^{\dagger} a\right)^{*}=\left(a^{\dagger} a b b^{\dagger}\right)^{*}=b b^{\dagger} a^{\dagger} a .
$$

Hence, the condition (c) holds.

(c) $\Rightarrow\left(\right.$ d): By the equality $a^{\dagger} a b b^{\dagger}=b b^{\dagger} a^{\dagger} a$, we get the condition (d):

$$
a^{\dagger} a\left(b b^{\dagger} a^{\dagger} a\right) b b^{\dagger}=a^{\dagger} a a^{\dagger} a b b^{\dagger} b b^{\dagger}=a^{\dagger} a b b^{\dagger}
$$

$(d) \Rightarrow(a)$ : Multiplying the assumption

$$
a^{\dagger} a b b^{\dagger}=a^{\dagger} a b b^{\dagger} a^{\dagger} a b b^{\dagger}
$$

by $a$ from the left side and by $b$ from the right side, we have that $a b=a b b^{\dagger} a^{\dagger} a b$. Therefore, the condition (a) is satisfied. 
(d) $\Leftrightarrow(\mathrm{e})$ : Applying the involution to $a^{\dagger} a b b^{\dagger}=a^{\dagger} a b b^{\dagger} a^{\dagger} a b b^{\dagger}$, we get $b b^{\dagger} a^{\dagger} a=$ $b b^{\dagger} a^{\dagger} a b b^{\dagger} a^{\dagger} a$. The opposite implication is analogous.

(b) $\Rightarrow$ (e): The condition $b^{\dagger} a^{\dagger} a b b^{\dagger} a^{\dagger}=b^{\dagger} a^{\dagger}$ implies

$$
b\left(b^{\dagger} a^{\dagger}\right) a=b b^{\dagger} a^{\dagger} a b b^{\dagger} a^{\dagger} a .
$$

(e) $\Rightarrow(b)$ : Multiplying the hypothesis

$$
b b^{\dagger} a^{\dagger} a=b b^{\dagger} a^{\dagger} a b b^{\dagger} a^{\dagger} a
$$

by $b^{\dagger}$ from the left side and by $a^{\dagger}$ from the right side, we get that the condition (b) holds: $b^{\dagger} a^{\dagger}=b^{\dagger} a^{\dagger} a b b^{\dagger} a^{\dagger}$.

(d) $\Rightarrow(\mathrm{g})$ : Assume that $a^{\dagger} a b b^{\dagger}=a^{\dagger} a b b^{\dagger} a^{\dagger} a b b^{\dagger}$. Then the condition (c) holds, so (g) follows trivially.

$(\mathrm{g}) \Rightarrow(\mathrm{d})$ : Using the assumption $\left(a^{\dagger} a b b^{\dagger}\right)^{\dagger}=b b^{\dagger} a^{\dagger} a$, we have

$$
a^{\dagger} a b b^{\dagger} a^{\dagger} a b b^{\dagger}=a^{\dagger} a b b^{\dagger}\left(b b^{\dagger} a^{\dagger} a\right) a^{\dagger} a b b^{\dagger}=a^{\dagger} a b b^{\dagger}\left(a^{\dagger} a b b^{\dagger}\right)^{\dagger} a^{\dagger} a b b^{\dagger}=a^{\dagger} a b b^{\dagger} .
$$

(g) $\Rightarrow$ (f): If $\left(a^{\dagger} a b b^{\dagger}\right)^{\dagger}=b b^{\dagger} a^{\dagger} a$, then

$$
b^{\dagger}\left(a^{\dagger} a b b^{\dagger}\right)^{\dagger} a^{\dagger}=b^{\dagger} b b^{\dagger} a^{\dagger} a a^{\dagger}=b^{\dagger} a^{\dagger} .
$$

(f) $\Rightarrow$ (g): Suppose that $b^{\dagger}\left(a^{\dagger} a b b^{\dagger}\right)^{\dagger} a^{\dagger}=b^{\dagger} a^{\dagger}$ holds. Then

$$
\begin{aligned}
& a^{\dagger} a b b^{\dagger}\left(b b^{\dagger} a^{\dagger} a\right) a^{\dagger} a b b^{\dagger}=a^{\dagger} a b\left(b^{\dagger} a^{\dagger}\right) a b b^{\dagger}=a^{\dagger} a b b^{\dagger}\left(a^{\dagger} a b b^{\dagger}\right)^{\dagger} a^{\dagger} a b b^{\dagger}=a^{\dagger} a b b^{\dagger}, \\
& b b^{\dagger} a^{\dagger} a\left(a^{\dagger} a b b^{\dagger}\right) b b^{\dagger} a^{\dagger} a=b\left(b^{\dagger} a^{\dagger}\right) a b\left(b^{\dagger} a^{\dagger}\right) a=b b^{\dagger}\left(a^{\dagger} a b b^{\dagger}\right)^{\dagger} a^{\dagger} a b b^{\dagger}\left(a^{\dagger} a b b^{\dagger}\right)^{\dagger} a^{\dagger} a \\
&=b\left(b^{\dagger}\left(a^{\dagger} a b b^{\dagger}\right)^{\dagger} a^{\dagger}\right) a=b b^{\dagger} a^{\dagger} a, \\
&\left(a^{\dagger} a b b^{\dagger} b b^{\dagger} a^{\dagger} a\right)^{*}=a^{\dagger} a b b^{\dagger} b b^{\dagger} a^{\dagger} a, \\
&\left(b b^{\dagger} a^{\dagger} a a^{\dagger} a b b^{\dagger}\right)^{*}=b b^{\dagger} a^{\dagger} a a^{\dagger} a b b^{\dagger} .
\end{aligned}
$$

Hence, $\left(a^{\dagger} a b b^{\dagger}\right)^{\dagger}=b b^{\dagger} a^{\dagger} a$ holds.

The second list of equivalent statements follows.

TheOrem 2.2. Let $\mathcal{A}$ be a unital $C^{*}$-algebra and let $a, b, a b \in \mathcal{A}^{-}$. Then the following conditions are equivalent:

(a) $a b(a b)^{\dagger}=a b b^{\dagger} a^{\dagger}$; 

(b) $a^{*} a b=b b^{\dagger} a^{*} a b$;
(c) $a^{*} a b b^{\dagger}=b b^{\dagger} a^{*} a$;
(d) $b^{\dagger} a^{\dagger} \in(a b)\{1,2,3\}$;
(e) $b^{\dagger} a^{\dagger} \in(a b)\{1,3\}$;
(f) $b\{1,3\} \cdot a\{1,3\} \subseteq(a b)\{1,3\}$;
(g) $a b(a b)^{\dagger} a=a b b^{\dagger}$;
(h) $\left(a b b^{\dagger}\right)^{\dagger}=b b^{\dagger} a^{\dagger}$;
(i) $b^{\dagger}\left(a b b^{\dagger}\right)^{\dagger}=b^{\dagger} a^{\dagger}$.

Proof. Observe that $a b \in \mathcal{A}^{-} \Leftrightarrow a b b^{\dagger} \in A^{-}$.

(a) $\Rightarrow(\mathrm{b})$ : Since $a b(a b)^{\dagger}=a b b^{\dagger} a^{\dagger}$ and $a b(a b)^{\dagger}$ is self-adjoint, then

$$
a b b^{\dagger} a^{\dagger}=\left(a b b^{\dagger} a^{\dagger}\right)^{*}=\left(a^{\dagger}\right)^{*} b b^{\dagger} a^{*}
$$

and

$$
a^{*} a b=a^{*} a b(a b)^{\dagger} a b=a^{*}\left(a b b^{\dagger} a^{\dagger}\right) a b=a^{*}\left(a^{\dagger}\right)^{*} b b^{\dagger} a^{*} a b=a^{\dagger} a b b^{\dagger} a^{*} a b .
$$

Using the hypothesis $a b(a b)^{\dagger}=a b b^{\dagger} a^{\dagger}$, we get $a b b^{\dagger} a^{\dagger} a b=a b(a b)^{\dagger} a b=a b$. This equality implies, by Theorem 2.1 (parts (a) and (c)), $a^{\dagger} a b b^{\dagger}=b b^{\dagger} a^{\dagger} a$. Now, from (2.3), we obtain

$$
a^{*} a b=\left(a^{\dagger} a b b^{\dagger}\right) a^{*} a b=b b^{\dagger} a^{\dagger} a a^{*} a b=b b^{\dagger} a^{*} a b .
$$

(b) $\Rightarrow$ (c): If $a^{*} a b=b b^{\dagger} a^{*} a b$, we have

$$
a^{*} a b b^{\dagger}=b b^{\dagger} a^{*} a b b^{\dagger}=\left(a b b^{\dagger}\right)^{*} a b b^{\dagger} .
$$

The right hand side of (2.4) is self-adjoint, which implies

$$
a^{*} a b b^{\dagger}=\left(a^{*} a b b^{\dagger}\right)^{*}=b b^{\dagger} a^{*} a .
$$

(c) $\Rightarrow$ (d): From $a^{*} a b b^{\dagger}=b b^{\dagger} a^{*} a$, we obtain

$$
a b b^{\dagger} a^{\dagger}=a a^{\dagger} a b b^{\dagger} a^{\dagger}=\left(a^{\dagger}\right)^{*}\left(a^{*} a b b^{\dagger}\right) a^{\dagger}=\left(a^{\dagger}\right)^{*} b b^{\dagger} a^{*} a a^{\dagger}=\left(a^{\dagger}\right)^{*} b b^{\dagger} a^{*} .
$$

Then

$$
\left(a b b^{\dagger} a^{\dagger}\right)^{*}=\left(\left(a^{\dagger}\right)^{*} b b^{\dagger} a^{*}\right)^{*}=a b b^{\dagger} a^{\dagger} .
$$

Using (2.5) and (c), we get

$$
\left(a b b^{\dagger} a^{\dagger}\right) a b=\left(a^{\dagger}\right)^{*}\left(b b^{\dagger} a^{*} a\right) b=\left(a^{\dagger}\right)^{*} a^{*} a b b^{\dagger} b=a b .
$$


Also,

$$
\begin{aligned}
b^{\dagger} a^{\dagger} a b b^{\dagger} a^{\dagger} & =b^{\dagger} a^{*}\left(a^{\dagger}\right)^{*} b b^{\dagger} a^{\dagger} a a^{\dagger}=b^{\dagger} a^{*}\left(\left(a^{\dagger}\right)^{*} b b^{\dagger} a^{*}\right)\left(a^{\dagger}\right)^{*} a^{\dagger} \\
& =b^{\dagger}\left(a^{*} a b b^{\dagger}\right) a^{\dagger}\left(a^{\dagger}\right)^{*} a^{\dagger}=b^{\dagger} b b^{\dagger} a^{*} a a^{\dagger}\left(a^{\dagger}\right)^{*} a^{\dagger} \\
& =b^{\dagger} a^{*}\left(a^{\dagger}\right)^{*} a^{\dagger}=b^{\dagger} a^{\dagger} .
\end{aligned}
$$

By (2.6), (2.7) and (2.8), we conclude that $b^{\dagger} a^{\dagger} \in(a b)\{1,2,3\}$.

$(\mathrm{d}) \Rightarrow(\mathrm{e})$ : This part is obvious.

(e) $\Rightarrow(\mathrm{f})$ : Suppose that $a^{(1,3)} \in a\{1,3\}$ and $b^{(1,3)} \in b\{1,3\}$. Notice that

$$
\begin{aligned}
a a^{(1,3)} & =\left(a a^{(1,3)}\right)^{*}=\left(a^{(1,3)}\right)^{*} a^{*}=\left(a^{(1,3)}\right)^{*}\left(a a^{\dagger} a\right)^{*} \\
& =\left(a^{(1,3)}\right)^{*} a^{*} a a^{\dagger}=a a^{(1,3)} a a^{\dagger}=a a^{\dagger}
\end{aligned}
$$

Since $a b b^{\dagger} a^{\dagger} a b=a b$, by Theorem 2.1 (parts (a) and (c)), we have $a^{\dagger} a b b^{\dagger}=b b^{\dagger} a^{\dagger} a$. From this equality and (2.9), we get

$$
a b b^{(1,3)} a^{(1,3)}=a\left(a^{\dagger} a b b^{\dagger}\right) a^{(1,3)}=a b b^{\dagger} a^{\dagger} a a^{(1,3)}=a b b^{\dagger} a^{\dagger} a a^{\dagger}=a b b^{\dagger} a^{\dagger}
$$

Because $b^{\dagger} a^{\dagger} \in(a b)\{3\}$, then the element $a b b^{\dagger} a^{\dagger}$ is self-adjoint and we deduce, from (2.10), that $a b b^{(1,3)} a^{(1,3)}$ is self-adjoint. So, $b^{(1,3)} a^{(1,3)} \in(a b)\{3\}$. Using the equality (2.10) and the assumption $b^{\dagger} a^{\dagger} \in(a b)\{1\}$, we get

$$
a b b^{(1,3)} a^{(1,3)} a b=a b b^{\dagger} a^{\dagger} a b=a b,
$$

i.e., $b^{(1,3)} a^{(1,3)} \in(a b)\{1\}$. Hence, the condition (f) holds.

(f) $\Rightarrow$ (a): Since $b^{\dagger} \in b\{1,3\}$ and $a^{\dagger} \in a\{1,3\}$, by the hypothesis $b\{1,3\} \cdot a\{1,3\} \subseteq$ $(a b)\{1,3\}$, it follows that $b^{\dagger} a^{\dagger} \in(a b)\{1,3\}$. This implies

$$
\begin{aligned}
a b(a b)^{\dagger} & =\left(a b(a b)^{\dagger}\right)^{*}=\left(a b b^{\dagger} a^{\dagger} a b(a b)^{\dagger}\right)^{*} \\
& =\left(a b(a b)^{\dagger}\right)^{*}\left(a b b^{\dagger} a^{\dagger}\right)^{*}=a b(a b)^{\dagger} a b b^{\dagger} a^{\dagger} \\
& =a b b^{\dagger} a^{\dagger} .
\end{aligned}
$$

(a) $\Rightarrow(\mathrm{g})$ : The equality $a b(a b)^{\dagger}=a b b^{\dagger} a^{\dagger}$ gives $a b b^{\dagger} a^{\dagger}=\left(a^{\dagger}\right)^{*} b b^{\dagger} a^{*}$ and (c), by the previous part of the proof. Now

$$
a b(a b)^{\dagger} a=\left(a b b^{\dagger} a^{\dagger}\right) a=\left(a^{\dagger}\right)^{*}\left(b b^{\dagger} a^{*} a\right)=\left(a^{\dagger}\right)^{*} a^{*} a b b^{\dagger}=a b b^{\dagger} .
$$

$(\mathrm{g}) \Rightarrow(\mathrm{b})$ : Applying $a b(a b)^{\dagger} a=a b b^{\dagger}$, we have

$$
a^{*} a b=a^{*} a b(a b)^{\dagger} a b=\left(a b(a b)^{\dagger} a\right)^{*} a b=\left(a b b^{\dagger}\right)^{*} a b=b b^{\dagger} a^{*} a b .
$$


(d) $\Rightarrow$ (h): Suppose that $b^{\dagger} a^{\dagger} \in(a b)\{1,2,3\}$. Then

$$
\begin{gathered}
a b b^{\dagger}\left(b b^{\dagger} a^{\dagger}\right) a b b^{\dagger}=\left(a b b^{\dagger} a^{\dagger} a b\right) b^{\dagger}=a b b^{\dagger}, \\
b b^{\dagger} a^{\dagger}\left(a b b^{\dagger}\right) b b^{\dagger} a^{\dagger}=b\left(b^{\dagger} a^{\dagger} a b b^{\dagger} a^{\dagger}\right)=b b^{\dagger} a^{\dagger}, \\
\left(a b b^{\dagger} b b^{\dagger} a^{\dagger}\right)^{*}=\left(a b b^{\dagger} a^{\dagger}\right)^{*}=a b b^{\dagger} a^{\dagger}, \\
\left(b b^{\dagger} a^{\dagger} a b b^{\dagger}\right)^{*}=b b^{\dagger} a^{\dagger} a b b^{\dagger} .
\end{gathered}
$$

Hence, $\left(a b b^{\dagger}\right)^{\dagger}=b b^{\dagger} a^{\dagger}$.

(h) $\Rightarrow(\mathrm{d})$ : The condition $\left(a b b^{\dagger}\right)^{\dagger}=b b^{\dagger} a^{\dagger}$ implies

$$
\begin{gathered}
a b b^{\dagger} a^{\dagger} a b=\left(a b b^{\dagger} b b^{\dagger} a^{\dagger} a b b^{\dagger}\right) b=a b b^{\dagger} b=a b, \\
b^{\dagger} a^{\dagger} a b b^{\dagger} a^{\dagger}=b^{\dagger}\left(b b^{\dagger} a^{\dagger} a b b^{\dagger} b b^{\dagger} a^{\dagger}\right)=b^{\dagger} b b^{\dagger} a^{\dagger}=b^{\dagger} a^{\dagger}, \\
\left(a b b^{\dagger} a^{\dagger}\right)^{*}=\left(a b b^{\dagger} b b^{\dagger} a^{\dagger}\right)^{*}=a b b^{\dagger} b b^{\dagger} a^{\dagger}=a b b^{\dagger} a^{\dagger} .
\end{gathered}
$$

Thus, $b^{\dagger} a^{\dagger} \in(a b)\{1,2,3\}$.

(h) $\Rightarrow(\mathrm{i})$ : Obvious.

(i) $\Rightarrow$ (h): Assume that $b^{\dagger}\left(a b b^{\dagger}\right)^{\dagger}=b^{\dagger} a^{\dagger}$ holds. Now, we have

$$
\begin{gathered}
a b b^{\dagger} b b^{\dagger} a^{\dagger} a b b^{\dagger}=a b\left(b^{\dagger} a^{\dagger}\right) a b b^{\dagger}=a b b^{\dagger}\left(a b b^{\dagger}\right)^{\dagger} a b b^{\dagger}=a b b^{\dagger}, \\
\begin{aligned}
b b^{\dagger} a^{\dagger} a b b^{\dagger} b b^{\dagger} a^{\dagger}= & b\left(b^{\dagger} a^{\dagger}\right) a b\left(b^{\dagger} a^{\dagger}\right)=b b^{\dagger}\left(a b b^{\dagger}\right)^{\dagger} a b b^{\dagger}\left(a b b^{\dagger}\right)^{\dagger} \\
= & b\left(b^{\dagger}\left(a b b^{\dagger}\right)^{\dagger}\right)=b b^{\dagger} a^{\dagger}, \\
\left(a b b^{\dagger} b b^{\dagger} a^{\dagger}\right)^{*}= & \left(a b\left(b^{\dagger} a^{\dagger}\right)\right)^{*}=\left(a b b^{\dagger}\left(a b b^{\dagger}\right)^{\dagger}\right)^{*}=a b b^{\dagger}\left(a b b^{\dagger}\right)^{\dagger} \\
= & a b b^{\dagger} a^{\dagger}=a b b^{\dagger} b b^{\dagger} a^{\dagger}, \\
& \left(b b^{\dagger} a^{\dagger} a b b^{\dagger}\right)^{*}=b b^{\dagger} a^{\dagger} a b b^{\dagger} .
\end{aligned}
\end{gathered}
$$

So, we deduce that $\left(a b b^{\dagger}\right)^{\dagger}=b b^{\dagger} a^{\dagger}$ holds.

Theorem 2.3. Let $\mathcal{A}$ be a unital $C^{*}$-algebra and let $a, b, a b \in \mathcal{A}^{-}$. Then the following conditions are equivalent: 

(a) $(a b)^{\dagger} a b=b^{\dagger} a^{\dagger} a b$;
(b) $a b b^{*}=a b b^{*} a^{\dagger} a$;
(c) $b b^{*} a^{\dagger} a=a^{\dagger} a b b^{*}$;
(d) $b^{\dagger} a^{\dagger} \in(a b)\{1,2,4\}$;
(e) $b^{\dagger} a^{\dagger} \in(a b)\{1,4\}$;
(f) $b\{1,4\} \cdot a\{1,4\} \subseteq(a b)\{1,4\}$;
(g) $b(a b)^{\dagger} a b=a^{\dagger} a b$;
(h) $\left(a^{\dagger} a b\right)^{\dagger}=b^{\dagger} a^{\dagger} a$;
(i) $\left(a^{\dagger} a b\right)^{\dagger} a^{\dagger}=b^{\dagger} a^{\dagger}$.

Proof. Notice that $a b \in \mathcal{A}^{-} \Leftrightarrow a^{\dagger} a b \in A^{-}$.

(a) $\Rightarrow(\mathrm{b})$ : Since $(a b)^{\dagger} a b=b^{\dagger} a^{\dagger} a b$ and $(a b)^{\dagger} a b$ is self-adjoint, it follows that

$$
b^{\dagger} a^{\dagger} a b=\left(b^{\dagger} a^{\dagger} a b\right)^{*}=b^{*} a^{\dagger} a\left(b^{\dagger}\right)^{*} .
$$

Then

$$
a b b^{*}=a b(a b)^{\dagger} a b b^{*}=a b\left(b^{\dagger} a^{\dagger} a b\right) b^{*}=a b b^{*} a^{\dagger} a\left(b^{\dagger}\right)^{*} b^{*}=a b b^{*} a^{\dagger} a b b^{\dagger} .
$$

Using the assumption $(a b)^{\dagger} a b=b^{\dagger} a^{\dagger} a b$, we obtain $a b b^{\dagger} a^{\dagger} a b=a b(a b)^{\dagger} a b=a b$. By Theorem 2.1 (parts (a) and (c)), this equality gives $a^{\dagger} a b b^{\dagger}=b b^{\dagger} a^{\dagger} a$. So, from (2.11), we get

$$
a b b^{*}=a b b^{*}\left(a^{\dagger} a b b^{\dagger}\right)=a b b^{*} b b^{\dagger} a^{\dagger} a=a b b^{*} a^{\dagger} a .
$$

(b) $\Rightarrow(\mathrm{c})$ : By the condition $a b b^{*}=a b b^{*} a^{\dagger} a$, we have

$$
a^{\dagger} a b b^{*}=a^{\dagger} a b b^{*} a^{\dagger} a=a^{\dagger} a b\left(a^{\dagger} a b\right)^{*} .
$$

The right hand side of (2.12) is self-adjoint, which gives

$$
a^{\dagger} a b b^{*}=\left(a^{\dagger} a b b^{*}\right)^{*}=b b^{*} a^{\dagger} a .
$$

(c) $\Rightarrow$ (d): The hypothesis $b b^{*} a^{\dagger} a=a^{\dagger} a b b^{*}$ gives

$$
b^{\dagger} a^{\dagger} a b=b^{\dagger} a^{\dagger} a b b^{\dagger} b=b^{\dagger}\left(a^{\dagger} a b b^{*}\right)\left(b^{\dagger}\right)^{*}=b^{\dagger} b b^{*} a^{\dagger} a\left(b^{\dagger}\right)^{*}=b^{*} a^{\dagger} a\left(b^{\dagger}\right)^{*}
$$

Now

$$
\left(b^{\dagger} a^{\dagger} a b\right)^{*}=\left(b^{*} a^{\dagger} a\left(b^{\dagger}\right)^{*}\right)^{*}=b^{\dagger} a^{\dagger} a b
$$

From the equalities (2.13) and (c), we obtain

$$
a b\left(b^{\dagger} a^{\dagger} a b\right)=a\left(b b^{*} a^{\dagger} a\right)\left(b^{\dagger}\right)^{*}=a a^{\dagger} a b b^{*}\left(b^{\dagger}\right)^{*}=a b
$$


and

$$
\begin{aligned}
b^{\dagger} a^{\dagger} a b b^{\dagger} a^{\dagger} & =b^{\dagger} b b^{\dagger} a^{\dagger} a\left(b^{\dagger}\right)^{*} b^{*} a^{\dagger}=b^{\dagger}\left(b^{\dagger}\right)^{*}\left(b^{*} a^{\dagger} a\left(b^{\dagger}\right)^{*}\right) b^{*} a^{\dagger} \\
& =b^{\dagger}\left(b^{\dagger}\right)^{*} b^{\dagger}\left(a^{\dagger} a b b^{*}\right) a^{\dagger}=b^{\dagger}\left(b^{\dagger}\right)^{*} b^{\dagger} b b^{*} a^{\dagger} a a^{\dagger} \\
& =b^{\dagger}\left(b^{\dagger}\right)^{*} b^{*} a^{\dagger}=b^{\dagger} a^{\dagger} .
\end{aligned}
$$

Hence, by (2.14), (2.15) and (2.16), it follows that $b^{\dagger} a^{\dagger} \in(a b)\{1,2,4\}$.

$(\mathrm{d}) \Rightarrow(\mathrm{e})$ : Obvious.

(e) $\Rightarrow$ (f): Assume that $a^{(1,4)} \in a\{1,4\}$ and $b^{(1,4)} \in b\{1,4\}$. Observe that

$$
\begin{aligned}
a^{(1,4)} a & =\left(a^{(1,4)} a\right)^{*}=a^{*}\left(a^{(1,4)}\right)^{*}=\left(a a^{\dagger} a\right)^{*}\left(a^{(1,4)}\right)^{*} \\
& =a^{\dagger} a a^{*}\left(a^{(1,4)}\right)^{*}=a^{\dagger} a a^{(1,4)} a=a^{\dagger} a .
\end{aligned}
$$

It is well known that the hypothesis $b^{\dagger} a^{\dagger} \in(a b)\{1\}$ implies $a^{\dagger} a b b^{\dagger}=b b^{\dagger} a^{\dagger} a$. By this and $(2.17)$, we obtain

$$
b^{(1,4)} a^{(1,4)} a b=b^{(1,4)}\left(a^{\dagger} a b b^{\dagger}\right) b=b^{(1,4)} b b^{\dagger} a^{\dagger} a b=b^{\dagger} b b^{\dagger} a^{\dagger} a b=b^{\dagger} a^{\dagger} a b .
$$

Now the condition $b^{\dagger} a^{\dagger} \in(a b)\{4\}$ implies that $b^{(1,4)} a^{(1,4)} \in(a b)\{4\}$. From the equality (2.18) and the assumption $b^{\dagger} a^{\dagger} \in(a b)\{1\}$, we get

$$
a b b^{(1,4)} a^{(1,4)} a b=a b b^{\dagger} a^{\dagger} a b=a b .
$$

Thus, $b^{(1,4)} a^{(1,4)} \in(a b)\{1\}$ and the condition (f) is satisfied.

(f) $\Rightarrow(\mathrm{a})$ : Because $b^{\dagger} \in b\{1,4\}$ and $a^{\dagger} \in a\{1,4\}$, from the assumption $b\{1,4\}$. $a\{1,4\} \subseteq(a b)\{1,4\}$, we get $b^{\dagger} a^{\dagger} \in(a b)\{1,4\}$. Then

$$
\begin{aligned}
(a b)^{\dagger} a b & =\left((a b)^{\dagger} a b\right)^{*}=\left((a b)^{\dagger} a b b^{\dagger} a^{\dagger} a b\right)^{*} \\
& =\left(b^{\dagger} a^{\dagger} a b\right)^{*}\left((a b)^{\dagger} a b\right)^{*}=b^{\dagger} a^{\dagger} a b(a b)^{\dagger} a b \\
& =b^{\dagger} a^{\dagger} a b .
\end{aligned}
$$

(a) $\Rightarrow(\mathrm{g})$ : The equality $(a b)^{\dagger} a b=b^{\dagger} a^{\dagger} a b$ implies $b^{\dagger} a^{\dagger} a b=b^{*} a^{\dagger} a\left(b^{\dagger}\right)^{*}$ and (c), by the previous part of the proof. Then

$$
b(a b)^{\dagger} a b=b\left(b^{\dagger} a^{\dagger} a b\right)=\left(b b^{*} a^{\dagger} a\right)\left(b^{\dagger}\right)^{*}=a^{\dagger} a b b^{*}\left(b^{\dagger}\right)^{*}=a^{\dagger} a b .
$$

$(\mathrm{g}) \Rightarrow(\mathrm{b})$ : From $b(a b)^{\dagger} a b=a^{\dagger} a b$, we obtian

$$
a b b^{*}=a b(a b)^{\dagger} a b b^{*}=a b\left(b(a b)^{\dagger} a b\right)^{*}=a b\left(a^{\dagger} a b\right)^{*}=a b b^{*} a^{\dagger} a .
$$

$(\mathrm{d}) \Rightarrow(\mathrm{h})$ : Assume that $b^{\dagger} a^{\dagger} \in(a b)\{1,2,4\}$. Now

$$
a^{\dagger} a b b^{\dagger} a^{\dagger} a a^{\dagger} a b=a^{\dagger}\left(a b b^{\dagger} a^{\dagger} a b\right)=a^{\dagger} a b
$$




$$
\begin{gathered}
b^{\dagger} a^{\dagger} a a^{\dagger} a b b^{\dagger} a^{\dagger} a=\left(b^{\dagger} a^{\dagger} a b b^{\dagger} a^{\dagger}\right) a=b^{\dagger} a^{\dagger} a, \\
\left(a^{\dagger} a b b^{\dagger} a^{\dagger} a\right)^{*}=a^{\dagger} a b b^{\dagger} a^{\dagger} a, \\
\left(b^{\dagger} a^{\dagger} a a^{\dagger} a b\right)^{*}=\left(b^{\dagger} a^{\dagger} a b\right)^{*}=b^{\dagger} a^{\dagger} a b=b^{\dagger} a^{\dagger} a a^{\dagger} a b .
\end{gathered}
$$

Thus, by definition, $\left(a^{\dagger} a b\right)^{\dagger}=b^{\dagger} a^{\dagger} a$.

(h) $\Rightarrow$ (d): If $\left(a^{\dagger} a b\right)^{\dagger}=b^{\dagger} a^{\dagger} a$, then

$$
\begin{gathered}
a b b^{\dagger} a^{\dagger} a b=a\left(a^{\dagger} a b b^{\dagger} a^{\dagger} a a^{\dagger} a b\right)=a a^{\dagger} a b=a b, \\
b^{\dagger} a^{\dagger} a b b^{\dagger} a^{\dagger}=\left(b^{\dagger} a^{\dagger} a a^{\dagger} a b b^{\dagger} a^{\dagger} a\right) a^{\dagger}=b^{\dagger} a^{\dagger} a a^{\dagger}=b^{\dagger} a^{\dagger}, \\
\left(b^{\dagger} a^{\dagger} a b\right)^{*}=\left(b^{\dagger} a^{\dagger} a a^{\dagger} a b\right)^{*}=b^{\dagger} a^{\dagger} a a^{\dagger} a b=b^{\dagger} a^{\dagger} a b .
\end{gathered}
$$

Therefore, $b^{\dagger} a^{\dagger} \in(a b)\{1,2,4\}$.

(h) $\Rightarrow$ (i): Obvious.

(i) $\Rightarrow$ (h): Applying the equality $\left(a^{\dagger} a b\right)^{\dagger} a^{\dagger}=b^{\dagger} a^{\dagger}$, we have

$$
\begin{aligned}
a^{\dagger} a b b^{\dagger} a^{\dagger} a a^{\dagger} a b= & a^{\dagger} a b\left(b^{\dagger} a^{\dagger}\right) a b=a^{\dagger} a b\left(a^{\dagger} a b\right)^{\dagger} a^{\dagger} a b=a^{\dagger} a b, \\
b^{\dagger} a^{\dagger} a a^{\dagger} a b b^{\dagger} a^{\dagger} a= & \left(b^{\dagger} a^{\dagger}\right) a b\left(b^{\dagger} a^{\dagger}\right) a=\left(a^{\dagger} a b\right)^{\dagger} a^{\dagger} a b\left(a^{\dagger} a b\right)^{\dagger} a^{\dagger} a \\
= & \left(\left(a^{\dagger} a b\right)^{\dagger} a^{\dagger}\right) a=b^{\dagger} a^{\dagger} a, \\
& \left(a^{\dagger} a b b^{\dagger} a^{\dagger} a\right)^{*}=a^{\dagger} a b b^{\dagger} a^{\dagger} a, \\
\left(b^{\dagger} a^{\dagger} a a^{\dagger} a b\right)^{*}= & \left(\left(b^{\dagger} a^{\dagger}\right) a b\right)^{*}=\left(\left(a^{\dagger} a b\right)^{\dagger} a^{\dagger} a b\right)^{*}=\left(\left(a^{\dagger} a b\right)^{\dagger} a^{\dagger}\right) a b \\
= & b^{\dagger} a^{\dagger} a b=b^{\dagger} a^{\dagger} a a^{\dagger} a b .
\end{aligned}
$$

Hence, $\left(a^{\dagger} a b\right)^{\dagger}=b^{\dagger} a^{\dagger} a$.

The combination of Theorem 2.2 and Theorem 2.3 yields a group of equivalent conditions for $(a b)^{\dagger}=b^{\dagger} a^{\dagger}$ to hold. Notice that the equivalences (c) and (d) of the following theorem appear in [9, Lemma 5].

Theorem 2.4. Let $\mathcal{A}$ be a unital $C^{*}$-algebra and let $a, b, a b \in \mathcal{A}^{-}$. Then the following conditions are equivalent:

(a) $(a b)^{\dagger}=b^{\dagger} a^{\dagger}$; 
(b) $a b(a b)^{\dagger}=a b b^{\dagger} a^{\dagger}$ and $(a b)^{\dagger} a b=b^{\dagger} a^{\dagger} a b$;

(c) $a^{*} a b=b b^{\dagger} a^{*} a b$ and $a b b^{*}=a b b^{*} a^{\dagger} a$;

(d) $a^{*} a b b^{\dagger}=b b^{\dagger} a^{*} a$ and $b b^{*} a^{\dagger} a=a^{\dagger} a b b^{*}$;

(e) $b^{\dagger} a^{\dagger} \in(a b)\{1,3,4\}$;

(f) $b\{1,3\} \cdot a\{1,3\} \subseteq(a b)\{1,3\}$ and $b\{1,4\} \cdot a\{1,4\} \subseteq(a b)\{1,4\}$;

(g) $a b(a b)^{\dagger} a=a b b^{\dagger}$ and $b(a b)^{\dagger} a b=a^{\dagger} a b$;

(h) $\left(a b b^{\dagger}\right)^{\dagger}=b b^{\dagger} a^{\dagger}$ and $\left(a^{\dagger} a b\right)^{\dagger}=b^{\dagger} a^{\dagger} a$;

(i) $b^{\dagger}\left(a b b^{\dagger}\right)^{\dagger}=b^{\dagger} a^{\dagger}$ and $\left(a^{\dagger} a b\right)^{\dagger} a^{\dagger}=b^{\dagger} a^{\dagger}$.

REMARK 2.5. The preceding theorems hold in rings with involution assuming

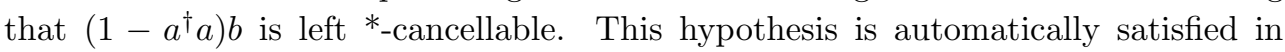
$C^{*}$ and *-reducing rings. Hence, we recover the results in $[4,5]$ for Hilbert space operators. The results of Tian [16] are obtained as a special case of our results.

In the following theorem, we prove another group of equivalent conditions for $(a b)^{\dagger}=b^{\dagger} a^{\dagger}$ to be satisfied.

Theorem 2.6. Let $\mathcal{A}$ be a unital $C^{*}$-algebra and let $a, b, a b \in \mathcal{A}^{-}$. Then $(a b)^{\dagger}=$ $b^{\dagger} a^{\dagger}$ if and only if $(a b)^{\dagger}=b^{\dagger}\left(a^{\dagger} a b b^{\dagger}\right)^{\dagger} a^{\dagger}$ and any one of the following equivalent conditions holds:

(a) $a b b^{\dagger} a^{\dagger} a b=a b$;

(b) $b^{\dagger} a^{\dagger} a b b^{\dagger} a^{\dagger}=b^{\dagger} a^{\dagger}$;

(c) $a^{\dagger} a b b^{\dagger}=b b^{\dagger} a^{\dagger} a$;

(d) $a^{\dagger} a b b^{\dagger}$ is an idempotent;

(e) $b b^{\dagger} a^{\dagger} a$ is an idempotent;

(f) $b^{\dagger}\left(a^{\dagger} a b b^{\dagger}\right)^{\dagger} a^{\dagger}=b^{\dagger} a^{\dagger}$;

(g) $\left(a^{\dagger} a b b^{\dagger}\right)^{\dagger}=b b^{\dagger} a^{\dagger} a$.

Proof. $\Longrightarrow$ : From the equality $(a b)^{\dagger}=b^{\dagger} a^{\dagger}$, we get $a b b^{\dagger} a^{\dagger} a b=a b$. Thus, by Theorem 2.1, the conditions (a)-(g) are satisfied and $(a b)^{\dagger}=b^{\dagger} a^{\dagger}=b^{\dagger}\left(a^{\dagger} a b b^{\dagger}\right)^{\dagger} a^{\dagger}$.

$\Longleftarrow$ : Conversely, the conditions (a)-(g) imply $b^{\dagger} a^{\dagger}=b^{\dagger}\left(a^{\dagger} a b b^{\dagger}\right)^{\dagger} a^{\dagger}$. Now the hypothesis $(a b)^{\dagger}=b^{\dagger}\left(a^{\dagger} a b b^{\dagger}\right)^{\dagger} a^{\dagger}$ gives $(a b)^{\dagger}=b^{\dagger} a^{\dagger}$.

The condition $(a b)^{\dagger}=b^{\dagger}\left(a^{\dagger} a b b^{\dagger}\right)^{\dagger} a^{\dagger}$ in Theorem 2.6 can be replaced by some equivalent conditions, as it can be seen in the following theorem.

Theorem 2.7. Let $\mathcal{A}$ be a unital $C^{*}$-algebra and let $a, b, a b \in \mathcal{A}^{-}$. Then the following statements are equivalent:
(a) $(a b)^{\dagger}=b^{\dagger}\left(a^{\dagger} a b b^{\dagger}\right)^{\dagger} a^{\dagger}$;
(b) $\left(a^{\dagger} a b b^{\dagger}\right)^{\dagger}=b(a b)^{\dagger} a$;
(c) $(a b)^{\dagger}=\left(a^{\dagger} a b\right)^{\dagger} a^{\dagger}=b^{\dagger}\left(a b b^{\dagger}\right)^{\dagger}$;
(d) $\left(a^{\dagger} a b\right)^{\dagger}=(a b)^{\dagger} a$ and $\left(a b b^{\dagger}\right)^{\dagger}=b(a b)^{\dagger}$; 
(e) $\left(a^{\dagger} a b\right)^{\dagger}=b^{\dagger}\left(a^{\dagger} a b b^{\dagger}\right)^{\dagger}$ and $\left(a b b^{\dagger}\right)^{\dagger}=\left(a^{\dagger} a b b^{\dagger}\right)^{\dagger} a^{\dagger}$;

(f) $(a b)^{\dagger}=b^{*}\left(a^{*} a b b^{*}\right)^{\dagger} a^{*}$;

(g) $\left(a^{*} a b b^{*}\right)^{\dagger}=\left(b^{*}\right)^{\dagger}(a b)^{\dagger}\left(a^{*}\right)^{\dagger}$.

Proof. We can easily see that $a b \in \mathcal{A}^{-} \Leftrightarrow a^{*} a b b^{*} \in A^{-}$.

(a) $\Rightarrow(\mathrm{e})$ : By the hypothesis $(a b)^{\dagger}=b^{\dagger}\left(a^{\dagger} a b b^{\dagger}\right)^{\dagger} a^{\dagger}$, we have

$$
\begin{gathered}
a^{\dagger} a b b^{\dagger}\left(a^{\dagger} a b b^{\dagger}\right)^{\dagger} a^{\dagger} a b=a^{\dagger} a b(a b)^{\dagger} a b=a^{\dagger} a b, \\
b^{\dagger}\left(a^{\dagger} a b b^{\dagger}\right)^{\dagger} a^{\dagger} a b b^{\dagger}\left(a^{\dagger} a b b^{\dagger}\right)^{\dagger}=b^{\dagger}\left(a^{\dagger} a b b^{\dagger}\right)^{\dagger}, \\
\left(a^{\dagger} a b b^{\dagger}\left(a^{\dagger} a b b^{\dagger}\right)^{\dagger}\right)^{*}=a^{\dagger} a b b^{\dagger}\left(a^{\dagger} a b b^{\dagger}\right)^{\dagger}, \\
\left(b^{\dagger}\left(a^{\dagger} a b b^{\dagger}\right)^{\dagger} a^{\dagger} a b\right)^{*}=\left((a b)^{\dagger} a b\right)^{*}=(a b)^{\dagger} a b=b^{\dagger}\left(a^{\dagger} a b b^{\dagger}\right)^{\dagger} a^{\dagger} a b .
\end{gathered}
$$

So, $\left(a^{\dagger} a b\right)^{\dagger}=b^{\dagger}\left(a^{\dagger} a b b^{\dagger}\right)^{\dagger}$.

Similarly

$$
\begin{gathered}
a b b^{\dagger}\left(a^{\dagger} a b b^{\dagger}\right)^{\dagger} a^{\dagger} a b b^{\dagger}=a b(a b)^{\dagger} a b b^{\dagger}=a b b^{\dagger}, \\
\left(a^{\dagger} a b b^{\dagger}\right)^{\dagger} a^{\dagger} a b b^{\dagger}\left(a^{\dagger} a b b^{\dagger}\right)^{\dagger} a^{\dagger}=\left(a^{\dagger} a b b^{\dagger}\right)^{\dagger} a^{\dagger}, \\
\left(a b b^{\dagger}\left(a^{\dagger} a b b^{\dagger}\right)^{\dagger} a^{\dagger}\right)^{*}=\left(a b(a b)^{\dagger}\right)^{*}=a b(a b)^{\dagger}=a b b^{\dagger}\left(a^{\dagger} a b b^{\dagger}\right)^{\dagger} a^{\dagger}, \\
\left(\left(a^{\dagger} a b b^{\dagger}\right)^{\dagger} a^{\dagger} a b b^{\dagger}\right)^{*}=\left(a^{\dagger} a b b^{\dagger}\right)^{\dagger} a^{\dagger} a b b^{\dagger} .
\end{gathered}
$$

Thus, $\left(a b b^{\dagger}\right)^{\dagger}=\left(a^{\dagger} a b b^{\dagger}\right)^{\dagger} a^{\dagger}$.

(e) $\Rightarrow(\mathrm{a})$ : The conditions $\left(a^{\dagger} a b\right)^{\dagger}=b^{\dagger}\left(a^{\dagger} a b b^{\dagger}\right)^{\dagger}$ and $\left(a b b^{\dagger}\right)^{\dagger}=\left(a^{\dagger} a b b^{\dagger}\right)^{\dagger} a^{\dagger}$ imply

$$
\begin{gathered}
a b b^{\dagger}\left(a^{\dagger} a b b^{\dagger}\right)^{\dagger} a^{\dagger} a b=a a^{\dagger} a b b^{\dagger}\left(a^{\dagger} a b b^{\dagger}\right)^{\dagger} a^{\dagger} a b b^{\dagger} b=a a^{\dagger} a b b^{\dagger} b=a b, \\
b^{\dagger}\left(a^{\dagger} a b b^{\dagger}\right)^{\dagger} a^{\dagger} a b b^{\dagger}\left(a^{\dagger} a b b^{\dagger}\right)^{\dagger} a^{\dagger}=b^{\dagger}\left(a^{\dagger} a b b^{\dagger}\right)^{\dagger} a^{\dagger} \\
\left(a b b^{\dagger}\left(a^{\dagger} a b b^{\dagger}\right)^{\dagger} a^{\dagger}\right)^{*}=a b b^{\dagger}\left(a^{\dagger} a b b^{\dagger}\right)^{\dagger} a^{\dagger} \\
\left(b^{\dagger}\left(a^{\dagger} a b b^{\dagger}\right)^{\dagger} a^{\dagger} a b\right)^{*}=b^{\dagger}\left(a^{\dagger} a b b^{\dagger}\right)^{\dagger} a^{\dagger} a b
\end{gathered}
$$

i.e., $(a b)^{\dagger}=b^{\dagger}\left(a^{\dagger} a b b^{\dagger}\right)^{\dagger} a^{\dagger}$. 
(b) $\Rightarrow$ (a): From the equality $\left(a^{\dagger} a b b^{\dagger}\right)^{\dagger}=b(a b)^{\dagger} a$, we get

$$
\begin{gathered}
a b b^{\dagger}\left(a^{\dagger} a b b^{\dagger}\right)^{\dagger} a^{\dagger} a b=a b b^{\dagger} b(a b)^{\dagger} a a^{\dagger} a b=a b(a b)^{\dagger} a b=a b, \\
\begin{aligned}
b^{\dagger}\left(a^{\dagger} a b b^{\dagger}\right)^{\dagger} a^{\dagger} a b b^{\dagger}\left(a^{\dagger} a b b^{\dagger}\right)^{\dagger} a^{\dagger}=b^{\dagger} b(a b)^{\dagger} a a^{\dagger} a b b^{\dagger} b(a b)^{\dagger} a a^{\dagger} \\
=b^{\dagger} b(a b)^{\dagger} a b(a b)^{\dagger} a a^{\dagger} \\
=b^{\dagger} b(a b)^{\dagger} a a^{\dagger} \\
=b^{\dagger}\left(a^{\dagger} a b b^{\dagger}\right)^{\dagger} a^{\dagger}, \\
\left(a b b^{\dagger}\left(a^{\dagger} a b b^{\dagger}\right)^{\dagger} a^{\dagger}\right)^{*}=\left(a b b^{\dagger} b(a b)^{\dagger} a a^{\dagger}\right)^{*}=\left(a b(a b)^{\dagger} a a^{\dagger}\right)^{*} \\
=a a^{\dagger} a b(a b)^{\dagger}=a b(a b)^{\dagger}=\left(a b(a b)^{\dagger}\right)^{*} \\
=\left(a a^{\dagger} a b(a b)^{\dagger}\right)^{*}=a b(a b)^{\dagger} a a^{\dagger} \\
=a b b^{\dagger} b(a b)^{\dagger} a a^{\dagger}=a b b^{\dagger}\left(a^{\dagger} a b b^{\dagger}\right)^{\dagger} a^{\dagger}, \\
\left(b^{\dagger}\left(a^{\dagger} a b b^{\dagger}\right)^{\dagger} a^{\dagger} a b\right)^{*}=\left(b^{\dagger} b(a b)^{\dagger} a a^{\dagger} a b\right)^{*}=\left(b^{\dagger} b(a b)^{\dagger} a b\right)^{*} \\
=(a b)^{\dagger} a b b^{\dagger} b=(a b)^{\dagger} a b=\left((a b)^{\dagger} a b\right)^{*} \\
=\left((a b)^{\dagger} a b b^{\dagger} b\right)^{*}=b^{\dagger} b(a b)^{\dagger} a b \\
=b^{\dagger} b(a b)^{\dagger} a a^{\dagger} a b=b^{\dagger}\left(a^{\dagger} a b b^{\dagger}\right)^{\dagger} a^{\dagger} a b .
\end{aligned}
\end{gathered}
$$

Therefore, $(a b)^{\dagger}=b^{\dagger}\left(a^{\dagger} a b b^{\dagger}\right)^{\dagger} a^{\dagger}$.

(a) $\Rightarrow$ (c): Assume that $(a b)^{\dagger}=b^{\dagger}\left(a^{\dagger} a b b^{\dagger}\right)^{\dagger} a^{\dagger}$ holds. Then the condition (e) holds and $(a b)^{\dagger}=\left(a^{\dagger} a b\right)^{\dagger} a^{\dagger}=b^{\dagger}\left(a b b^{\dagger}\right)^{\dagger}$.

(c) $\Rightarrow$ (d): The hypothesis $(a b)^{\dagger}=\left(a^{\dagger} a b\right)^{\dagger} a^{\dagger}$ implies

$$
\begin{aligned}
& a^{\dagger} a b(a b)^{\dagger} a a^{\dagger} a b=a^{\dagger} a b(a b)^{\dagger} a b=a^{\dagger} a b, \\
& (a b)^{\dagger} a a^{\dagger} a b(a b)^{\dagger} a=(a b)^{\dagger} a b(a b)^{\dagger} a=(a b)^{\dagger} a, \\
& \left(a^{\dagger} a b(a b)^{\dagger} a\right)^{*}=\left(a^{\dagger} a b\left(a^{\dagger} a b\right)^{\dagger} a^{\dagger} a\right)^{*}=a^{\dagger} a a^{\dagger} a b\left(a^{\dagger} a b\right)^{\dagger} \\
& =a^{\dagger} a b\left(a^{\dagger} a b\right)^{\dagger}=\left(a^{\dagger} a b\left(a^{\dagger} a b\right)^{\dagger}\right)^{*} \\
& =\left(a^{\dagger} a a^{\dagger} a b\left(a^{\dagger} a b\right)^{\dagger}\right)^{*}=a^{\dagger} a b\left(a^{\dagger} a b\right)^{\dagger} a^{\dagger} a \\
& =a^{\dagger} a b(a b)^{\dagger} a \text {, } \\
& \left((a b)^{\dagger} a a^{\dagger} a b\right)^{*}=\left((a b)^{\dagger} a b\right)^{*}=(a b)^{\dagger} a b=(a b)^{\dagger} a a^{\dagger} a b .
\end{aligned}
$$

Thus, $\left(a^{\dagger} a b\right)^{\dagger}=(a b)^{\dagger} a$. By an analogy, from $(a b)^{\dagger}=b^{\dagger}\left(a b b^{\dagger}\right)^{\dagger}$, we obtain

$$
a b b^{\dagger} b(a b)^{\dagger} a b b^{\dagger}=a b(a b)^{\dagger} a b b^{\dagger}=a b b^{\dagger},
$$




$$
\begin{aligned}
& b(a b)^{\dagger} a b b^{\dagger} b(a b)^{\dagger}=b(a b)^{\dagger} a b(a b)^{\dagger}=b(a b)^{\dagger} \\
&\left(a b b^{\dagger} b(a b)^{\dagger}\right)^{*}=\left(a b(a b)^{\dagger}\right)^{*}=a b(a b)^{\dagger}=a b b^{\dagger} b(a b)^{\dagger} \\
&\left(b(a b)^{\dagger} a b b^{\dagger}\right)^{*}=\left(b b^{\dagger}\left(a b b^{\dagger}\right)^{\dagger} a b b^{\dagger}\right)^{*}=\left(a b b^{\dagger}\right)^{\dagger} a b b^{\dagger} b b^{\dagger} \\
&=\left(a b b^{\dagger}\right)^{\dagger} a b b^{\dagger}=\left(\left(a b b^{\dagger}\right)^{\dagger} a b b^{\dagger}\right)^{*} \\
&=\left(\left(a b b^{\dagger}\right)^{\dagger} a b b^{\dagger} b b^{\dagger}\right)^{*}=b b^{\dagger}\left(a b b^{\dagger}\right)^{\dagger} a b b^{\dagger} \\
&=b(a b)^{\dagger} a b b^{\dagger} .
\end{aligned}
$$

So, we deduce that $\left(a b b^{\dagger}\right)^{\dagger}=b(a b)^{\dagger}$.

$(\mathrm{d}) \Rightarrow(\mathrm{g})$ : Suppose that $\left(a^{\dagger} a b\right)^{\dagger}=(a b)^{\dagger} a$ and $\left(a b b^{\dagger}\right)^{\dagger}=b(a b)^{\dagger}$. Now,

$$
\begin{aligned}
a^{*} a b b^{*}\left(b^{*}\right)^{\dagger}(a b)^{\dagger}\left(a^{*}\right)^{\dagger} a^{*} a b b^{*} & =a^{*} a b b^{\dagger} b(a b)^{\dagger} a a^{\dagger} a b b^{*} \\
& =a^{*} a b(a b)^{\dagger} a b b^{*} \\
& =a^{*} a b b^{*} \\
\left(b^{*}\right)^{\dagger}(a b)^{\dagger}\left(a^{*}\right)^{\dagger} a^{*} a b b^{*}\left(b^{*}\right)^{\dagger}(a b)^{\dagger}\left(a^{*}\right)^{\dagger} & =\left(b^{*}\right)^{\dagger}(a b)^{\dagger} a a^{\dagger} a b b^{\dagger} b(a b)^{\dagger}\left(a^{*}\right)^{\dagger} \\
& =\left(b^{*}\right)^{\dagger}(a b)^{\dagger} a b(a b)^{\dagger}\left(a^{*}\right)^{\dagger} \\
& =\left(b^{*}\right)^{\dagger}(a b)^{\dagger}\left(a^{*}\right)^{\dagger} \\
\left(a^{*} a b b^{*}\left(b^{*}\right)^{\dagger}(a b)^{\dagger}\left(a^{*}\right)^{\dagger}\right)^{*}= & \left(a^{*} a b b^{\dagger} b(a b)^{\dagger}\left(a^{*}\right)^{\dagger}\right)^{*}=\left(a^{*} a b(a b)^{\dagger}\left(a^{*}\right)^{\dagger}\right)^{*} \\
& =a^{\dagger} a b(a b)^{\dagger} a=\left(a^{\dagger} a b(a b)^{\dagger} a\right)^{*} \\
& =a^{*} a b(a b)^{\dagger}\left(a^{*}\right)^{\dagger}=a^{*} a b b^{\dagger} b(a b)^{\dagger}\left(a^{*}\right)^{\dagger} \\
& =a^{*} a b b^{*}\left(b^{*}\right)^{\dagger}(a b)^{\dagger}\left(a^{*}\right)^{\dagger}, \\
\left(\left(b^{*}\right)^{\dagger}(a b)^{\dagger}\left(a^{*}\right)^{\dagger} a^{*} a b b^{*}\right)^{*} & =\left(\left(b^{*}\right)^{\dagger}(a b)^{\dagger} a a^{\dagger} a b b^{*}\right)^{*}=\left(\left(b^{*}\right)^{\dagger}(a b)^{\dagger} a b b^{*}\right)^{*} \\
& =b(a b)^{\dagger} a b b^{\dagger}=\left(b(a b)^{\dagger} a b b^{\dagger}\right)^{*} \\
& =\left(b^{*}\right)^{\dagger}(a b)^{\dagger} a b b^{*}=\left(b^{*}\right)^{\dagger}(a b)^{\dagger} a a^{\dagger} a b b^{*} \\
& =\left(b^{*}\right)^{\dagger}(a b)^{\dagger}\left(a^{*}\right)^{\dagger} a^{*} a b b^{*}
\end{aligned}
$$

Therefore, $\left(a^{*} a b b^{*}\right)^{\dagger}=\left(b^{*}\right)^{\dagger}(a b)^{\dagger}\left(a^{*}\right)^{\dagger}$.

$(\mathrm{g}) \Rightarrow(\mathrm{f})$ : From the condition $\left(a^{*} a b b^{*}\right)^{\dagger}=\left(b^{*}\right)^{\dagger}(a b)^{\dagger}\left(a^{*}\right)^{\dagger}$, we get $b^{*}\left(a^{*} a b b^{*}\right)^{\dagger} a^{*}=b^{*}\left(b^{*}\right)^{\dagger}(a b)^{\dagger}\left(a^{*}\right)^{\dagger} a^{*}=b^{\dagger} b(a b)^{\dagger} a a^{\dagger}$. Then, in the same way as in the proof of $(\mathrm{b}) \Rightarrow(\mathrm{a})$, we conclude $(a b)^{\dagger}=b^{*}\left(a^{*} a b b^{*}\right)^{\dagger} a^{*}$.

(f) $\Rightarrow(\mathrm{b})$ : If $(a b)^{\dagger}=b^{*}\left(a^{*} a b b^{*}\right)^{\dagger} a^{*}$, then

$$
a^{\dagger} a b b^{\dagger} b(a b)^{\dagger} a a^{\dagger} a b b^{\dagger}=a^{\dagger} a b(a b)^{\dagger} a b b^{\dagger}=a^{\dagger} a b b^{\dagger},
$$




$$
\begin{aligned}
& b(a b)^{\dagger} a a^{\dagger} a b b^{\dagger} b(a b)^{\dagger} a=b(a b)^{\dagger} a b(a b)^{\dagger} a=b(a b)^{\dagger} a \\
&\left(a^{\dagger} a b b^{\dagger} b(a b)^{\dagger} a\right)^{*}=\left(a^{\dagger} a b(a b)^{\dagger} a\right)^{*}=a^{*} a b(a b)^{\dagger}\left(a^{\dagger}\right)^{*} \\
&=a^{*} a b b^{*}\left(a^{*} a b b^{*}\right)^{\dagger} a^{*}\left(a^{\dagger}\right)^{*}=\left(a^{\dagger} a a^{*} a b b^{*}\left(a^{*} a b b^{*}\right)^{\dagger}\right)^{*} \\
&=\left(a^{*} a b b^{*}\left(a^{*} a b b^{*}\right)^{\dagger}\right)^{*}=a^{*} a b b^{*}\left(a^{*} a b b^{*}\right)^{\dagger} \\
&=a^{\dagger} a a^{*} a b b^{*}\left(a^{*} a b b^{*}\right)^{\dagger}=\left(a^{*} a b b^{*}\left(a^{*} a b b^{*}\right)^{\dagger} a^{*}\left(a^{\dagger}\right)^{*}\right)^{*} \\
&=\left(a^{*} a b(a b)^{\dagger}\left(a^{\dagger}\right)^{*}\right)^{*}=a^{\dagger} a b(a b)^{\dagger} a \\
&=a^{\dagger} a b b^{\dagger} b(a b)^{\dagger} a \\
&\left(b(a b)^{\dagger} a a^{\dagger} a b b^{\dagger}\right)^{*}=\left(b(a b)^{\dagger} a b b^{\dagger}\right)^{*}=\left(b^{\dagger}\right)^{*}(a b)^{\dagger} a b b^{*} \\
&=\left(b^{\dagger}\right)^{*} b^{*}\left(a^{*} a b b^{*}\right)^{\dagger} a^{*} a b b^{*}=\left(\left(a^{*} a b b^{*}\right)^{\dagger} a^{*} a b b^{*} b b^{\dagger}\right)^{*} \\
&=\left(\left(a^{*} a b b^{*}\right)^{\dagger} a^{*} a b b^{*}\right)^{*}=\left(a^{*} a b b^{*}\right)^{\dagger} a^{*} a b b^{*} \\
&=\left(a^{*} a b b^{*}\right)^{\dagger} a^{*} a b b^{*} b b^{\dagger}=\left(\left(b^{\dagger}\right)^{*} b^{*}\left(a^{*} a b b^{*}\right)^{\dagger} a^{*} a b b^{*}\right)^{*} \\
&=\left(\left(b^{\dagger}\right)^{*}(a b)^{\dagger} a b b^{*}\right)^{*}=b(a b)^{\dagger} a b b^{\dagger} \\
&=b(a b)^{\dagger} a a^{\dagger} a b b^{\dagger} .
\end{aligned}
$$

Hence, $\left(a^{\dagger} a b b^{\dagger}\right)^{\dagger}=b(a b)^{\dagger} a$.

3. Reverse order law for the weighted MP-inverse. We can also consider reverse order law for the weighted Moore-Penrose inverse. Based on the results in Section 2, we now can establish various equivalent conditions related to the weighted MP-inverse of a product of elements in $C^{*}$-algebra.

Corollary 3.1. Let $\mathcal{A}$ be a unital $C^{*}$-algebra and let e, $f, h$ be positive invertible elements of $\mathcal{A}$. If $a, b \in \mathcal{A}$ are regular, then the following conditions are equivalent:

(a) $a b b_{e, f}^{\dagger} a_{f, h}^{\dagger} a b=a b$;

(b) $b_{e, f}^{\dagger} a_{f, h}^{\dagger} a b b_{e, f}^{\dagger} a_{f, h}^{\dagger}=b_{e, f}^{\dagger} a_{f, h}^{\dagger}$;

(c) $a_{f, h}^{\dagger} a b b_{e, f}^{\dagger}=b b_{e, f}^{\dagger} a_{f, h}^{\dagger} a$;

(d) $a_{f, h}^{\dagger} a b b_{e, f}^{\dagger}$ is an idempotent;

(e) $b b_{e, f}^{\dagger} a_{f, h}^{\dagger} a$ is an idempotent;

(f) $b_{e, f}^{\dagger}\left(a_{f, h}^{\dagger} a b b_{e, f}^{\dagger}\right)_{f, f}^{\dagger} a_{f, h}^{\dagger}=b_{e, f}^{\dagger} a_{f, h}^{\dagger}$;

(g) $\left(a_{f, h}^{\dagger} a b b_{e, f}^{\dagger}\right)_{f, f}^{\dagger}=b b_{e, f}^{\dagger} a_{f, h}^{\dagger} a$.

Proof. (a) $\Leftrightarrow(\mathrm{b})$ : Suppose that $a_{1}=h^{1 / 2} a f^{-1 / 2}$ and $b_{1}=f^{1 / 2} b e^{-1 / 2}$. Then $a_{1} b_{1}=h^{1 / 2} a b e^{-1 / 2}$ and $a_{1}, b_{1}$ are regular if and only if $a, b$ are regular, respectively. From Theorem 1.4, we have $a_{f, h}^{\dagger}=f^{-1 / 2}\left(h^{1 / 2} a f^{-1 / 2}\right)^{\dagger} h^{1 / 2}=f^{-1 / 2} a_{1}^{\dagger} h^{1 / 2}$ and $b_{e, f}^{\dagger}=$ $e^{-1 / 2}\left(f^{1 / 2} b e^{-1 / 2}\right)^{\dagger} f^{1 / 2}=e^{-1 / 2} b_{1}^{\dagger} f^{1 / 2}$. 
It is easy to verify that the condition $a b b_{e, f}^{\dagger} a_{f, h}^{\dagger} a b=a b$ holds if and only if $a_{1} b_{1} b_{1}^{\dagger} a_{1}^{\dagger} a_{1} b_{1}=a_{1} b_{1}$. By Theorem 2.1, this is necessary and sufficient condition for the equality $b_{1}^{\dagger} a_{1}^{\dagger} a_{1} b_{1} b_{1}^{\dagger} a_{1}^{\dagger}=b_{1}^{\dagger} a_{1}^{\dagger}$ which is equivalent to $b_{e, f}^{\dagger} a_{f, h}^{\dagger} a b b_{e, f}^{\dagger} a_{f, h}^{\dagger}=b_{e, f}^{\dagger} a_{f, h}^{\dagger}$.

The rest of the proof follows analogously. We only mention that $a_{f, h}^{\dagger} a b b_{e, f}^{\dagger}$ is regular $\Leftrightarrow f^{-1 / 2} a_{1}^{\dagger} a_{1} b_{1} b_{1}^{\dagger} f^{1 / 2}$ is regular $\Leftrightarrow a_{1}^{\dagger} a_{1} b_{1} b_{1}$ is regular and, by Theorem 1.4, $\left(a_{f, h}^{\dagger} a b b_{e, f}^{\dagger}\right)_{f, f}^{\dagger}=\left(f^{-1 / 2} a_{1}^{\dagger} a_{1} b_{1} b_{1}^{\dagger} f^{1 / 2}\right)_{f, f}^{\dagger}=f^{-1 / 2}\left(a_{1}^{\dagger} a_{1} b_{1} b_{1}^{\dagger}\right)^{\dagger} f^{1 / 2}$.

In order to prove Corollary 3.1 (and other corollaries in this section) in ring with involution, we need to assume that $\left(1-a_{f, h}^{\dagger} a\right) b$ is left ${ }^{*} e, f$-cancellable where the function ${ }^{*} e, f$ is not general an involution. Hence, it is not enough to assume that an element is just cancellable.

Let $e, f$ be positive invertible elements of a unital $C^{*}$-algebra $\mathcal{A}$ and define $x^{* e, f}=$ $e^{-1} x^{*} f$.

Corollary 3.2. Let $\mathcal{A}$ be a unital $C^{*}$-algebra and let e, $f, h$ be positive invertible elements of $\mathcal{A}$. If $a, b, a b \in \mathcal{A}$ are regular, then the following conditions are equivalent:

(a) $a b(a b)_{e, h}^{\dagger}=a b b_{e, f}^{\dagger} a_{f, h}^{\dagger}$;

(b) $a^{* f, h} a b=b b_{e, f}^{\dagger} a^{* f, h} a b$;

(c) $a^{* f, h} a b b_{e, f}^{\dagger}=b b_{e, f}^{\dagger} a^{* f, h} a$;

(d) $b_{e, f}^{\dagger} a_{f, h}^{\dagger} \in(a b)\{1,2,4 h\}$;

(e) $b_{e, f}^{\dagger} a_{f, h}^{\dagger} \in(a b)\{1,4 h\}$;

(f) $b\{1,4 f\} \cdot a\{1,4 h\} \subseteq(a b)\{1,4 h\}$;

(g) $a b(a b)_{e, h}^{\dagger} a=a b b_{e, f}^{\dagger}$;

(h) $\left(a b b_{e, f}^{\dagger}\right)_{f, h}^{\dagger}=b b_{e, f}^{\dagger} a_{f, h}^{\dagger}$;

(i) $b_{e, f}^{\dagger}\left(a b b_{e, f}^{\dagger}\right)_{f, h}^{\dagger}=b_{e, f}^{\dagger} a_{f, h}^{\dagger}$.

Proof. (a) $\Leftrightarrow(\mathrm{b})$ : Let $a_{1}=h^{1 / 2} a f^{-1 / 2}$ and $b_{1}=f^{1 / 2} b e^{-1 / 2}$ as in the previous corollary. Thus, $a_{1} b_{1}=h^{1 / 2} a b e^{-1 / 2}$ and $a_{1}, b_{1}, a_{1} b_{1}$ are regular if and only if $a, b$, $a b$ are regular, respectively. By Theorem 1.4, we get $(a b)_{e, h}^{\dagger}=e^{-1 / 2}\left(a_{1} b_{1}\right)^{\dagger} h^{1 / 2}$ and $b_{e, f}^{\dagger} a_{f, h}^{\dagger}=e^{-1 / 2} b_{1}^{\dagger} a_{1}^{\dagger} h^{1 / 2}$.

Now, by Theorem 2.2,

$$
\begin{aligned}
a b(a b)_{e, h}^{\dagger}=a b b_{e, f}^{\dagger} a_{f, h}^{\dagger} & \Leftrightarrow a_{1} b_{1}\left(a_{1} b_{1}\right)^{\dagger}=a_{1} b_{1} b_{1}^{\dagger} a_{1}^{\dagger} \\
& \Leftrightarrow a_{1}^{*} a_{1} b_{1}=b_{1} b_{1}^{\dagger} a_{1}^{*} a_{1} b_{1} \\
& \Leftrightarrow a^{* f, h} a b=b b_{e, f}^{\dagger} a^{* f, h} a b .
\end{aligned}
$$

The rest of the proof follows analogously.

Corollary 3.3. Let $\mathcal{A}$ be a unital $C^{*}$-algebra and let e, $f, h$ be positive invertible 
elements of $\mathcal{A}$. If $a, b, a b \in \mathcal{A}$ are regular, then the following conditions are equivalent:
(a) $(a b)_{e, h}^{\dagger} a b=b_{e, f}^{\dagger} a_{f, h}^{\dagger} a b$;
(b) $a b b^{* e, f}=a b b^{* e, f} a_{f, h}^{\dagger} a$;
(c) $b b^{* e, f} a_{f, h}^{\dagger} a=a_{f, h}^{\dagger} a b b^{* e, f}$;
(d) $b_{e, f}^{\dagger} a_{f, h}^{\dagger} \in(a b)\{1,2,3 e\}$;
(e) $b_{e, f}^{\dagger} a_{f, h}^{\dagger} \in(a b)\{1,3 e\}$;
(f) $b\{1,3 e\} \cdot a\{1,3 f\} \subseteq(a b)\{1,3 e\}$;
(g) $b(a b)_{e, h}^{\dagger} a b=a_{f, h}^{\dagger} a b$;
(h) $\left(a_{f, h}^{\dagger} a b\right)_{e, f}^{\dagger}=b_{e, f}^{\dagger} a_{f, h}^{\dagger} a$;
(i) $\left(a_{f, h}^{\dagger} a b\right)_{e, f}^{\dagger} a_{f, h}^{\dagger}=b_{e, f}^{\dagger} a_{f, h}^{\dagger}$.

Proof. Let $a_{1}$ and $b_{1}$ be the same as in the previous corollary. For conditions (a)(g) we can find the equivalent expressions in terms of $a_{1}$ and $b_{1}$, and apply Theorem 2.3 to finish the proof.

The combination of Corollary 3.2 and Corollary 3.3 gives a list of equivalent conditions for $(a b)_{e, h}^{\dagger}=b_{e, f}^{\dagger} a_{f, h}^{\dagger}$ to hold.

Corollary 3.4. Let $\mathcal{A}$ be a unital $C^{*}$-algebra and let e, $f, h$ be positive invertible elements of $\mathcal{A}$. If $a, b, a b \in \mathcal{A}$ are regular, then the following conditions are equivalent:
(a) $(a b)_{e, h}^{\dagger}=b_{e, f}^{\dagger} a_{f, h}^{\dagger}$;
(b) $a b(a b)_{e, h}^{\dagger}=a b b_{e, f}^{\dagger} a_{f, h}^{\dagger}$ and $(a b)_{e, h}^{\dagger} a b=b_{e, f}^{\dagger} a_{f, h}^{\dagger} a b$;
(c) $a^{* f, h} a b=b b_{e, f}^{\dagger} a^{* f, h} a b$ and $a b b^{* e, f}=a b b^{* e, f} a_{f, h}^{\dagger} a$;
(d) $a^{* f, h} a b b_{e, f}^{\dagger}=b b_{e, f}^{\dagger} a^{* f, h} a$ and $b b^{* e, f} a_{f, h}^{\dagger} a=a_{f, h}^{\dagger} a b b^{* e, f}$;
(e) $b_{e, f}^{\dagger} a_{f, h}^{\dagger} \in(a b)\{1,3 e, 4 h\}$;
(f) $b\{1,3 e\} \cdot a\{1,3 f\} \subseteq(a b)\{1,3 e\}$ and $b\{1,4 f\} \cdot a\{1,4 h\} \subseteq(a b)\{1,4 h\}$;
(g) $a b(a b)_{e, h}^{\dagger} a=a b b_{e, f}^{\dagger}$ and $b(a b)_{e, h}^{\dagger} a b=a_{f, h}^{\dagger} a b$;
(h) $\left(a b b_{e, f}^{\dagger}\right)_{f, h}^{\dagger}=b b_{e, f}^{\dagger} a_{f, h}^{\dagger}$ and $\left(a_{f, h}^{\dagger} a b\right)_{e, f}^{\dagger}=b_{e, f}^{\dagger} a_{f, h}^{\dagger} a$;
(i) $b_{e, f}^{\dagger}\left(a b b_{e, f}^{\dagger}\right)_{f, h}^{\dagger}=b_{e, f}^{\dagger} a_{f, h}^{\dagger}$ and $\left(a_{f, h}^{\dagger} a b\right)_{e, f}^{\dagger} a_{f, h}^{\dagger}=b_{e, f}^{\dagger} a_{f, h}^{\dagger}$.

Corollary 3.5. Let $\mathcal{A}$ be a unital $C^{*}$-algebra and let e, $f, h$ be positive invertible elements of $\mathcal{A}$. If $a, b, a b \in \mathcal{A}$ are regular, then the following statements are equivalent:
(a) $(a b)_{e, h}^{\dagger}=b_{e, f}^{\dagger}\left(a_{f, h}^{\dagger} a b b_{e, f}^{\dagger}\right)_{f, f}^{\dagger} a_{f, h}^{\dagger}$;
(b) $\left(a_{f, h}^{\dagger} a b b_{e, f}^{\dagger}\right)_{f, f}^{\dagger}=b(a b)_{e, h}^{\dagger} a$;
(c) $(a b)_{e, h}^{\dagger}=\left(a_{f, h}^{\dagger} a b\right)_{e, f}^{\dagger} a_{f, h}^{\dagger}=b_{e, f}^{\dagger}\left(a b b_{e, f}^{\dagger}\right)_{f, h}^{\dagger}$;
(d) $\left(a_{f, h}^{\dagger} a b\right)_{e, f}^{\dagger}=(a b)_{e, h}^{\dagger} a$ and $\left(a b b_{e, f}^{\dagger}\right)_{f, h}^{\dagger}=b(a b)_{e, h}^{\dagger}$;
(e) $\left(a_{f, h}^{\dagger} a b\right)_{e, f}^{\dagger}=b_{e, f}^{\dagger}\left(a_{f, h}^{\dagger} a b b_{e, f}^{\dagger}\right)_{f, f}^{\dagger}$ and $\left(a b b_{e, f}^{\dagger}\right)_{f, h}^{\dagger}=\left(a_{f, h}^{\dagger} a b b_{e, f}^{\dagger}\right)_{f, f}^{\dagger} a_{f, h}^{\dagger}$;
(f) $(a b)_{e, h}^{\dagger}=b^{* e, f}\left(a^{* f, h} a b b^{* e, f}\right)_{f, f}^{\dagger} a^{* f, h}$; 
(g) $\left(a^{* f, h} a b b^{* e, f}\right)_{f, f}^{\dagger}=\left(b^{\dagger}\right)^{* f, e}(a b)_{e, h}^{\dagger}\left(a^{\dagger}\right)^{* h, f}$.

Proof. Let $a_{1}$ and $b_{1}$ be the same as in Corollary 3.1. Applying Theorem 2.7 to the equivalent expressions, in terms of $a_{1}$ and $b_{1}$, for conditions (a)-(g) we prove this corollary.

In the next result, we present again some equivalent conditions for the reverse order rule for the weighted Moore-Penrose inverse.

Corollary 3.6. Let $\mathcal{A}$ be a unital $C^{*}$-algebra and let e, $f, h$ be positive invertible elements of $\mathcal{A}$. If $a, b, a b \in \mathcal{A}$ are regular, then $(a b)_{e, h}^{\dagger}=b_{e, f}^{\dagger} a_{f, h}^{\dagger}$ if and only if $(a b)_{e, h}^{\dagger}=b_{e, f}^{\dagger}\left(a_{f, h}^{\dagger} a b b_{e, f}^{\dagger}\right)_{f, f}^{\dagger} a_{f, h}^{\dagger}$ and any one of the following equivalent conditions holds::

(a) $a b b_{e, f}^{\dagger} a_{f, h}^{\dagger} a b=a b$;

(b) $b_{e, f}^{\dagger} a_{f, h}^{\dagger} a b b_{e, f}^{\dagger} a_{f, h}^{\dagger}=b_{e, f}^{\dagger} a_{f, h}^{\dagger}$;

(c) $a_{f, h}^{\dagger} a b b_{e, f}^{\dagger}=b b_{e, f}^{\dagger} a_{f, h}^{\dagger} a$;

(d) $a_{f, h}^{\dagger} a b b_{e, f}^{\dagger}$ is an idempotent;

(e) $b b_{e, f}^{\dagger} a_{f, h}^{\dagger} a$ is an idempotent;

(f) $b_{e, f}^{\dagger}\left(a_{f, h}^{\dagger} a b b_{e, f}^{\dagger}\right)_{f, f}^{\dagger} a_{f, h}^{\dagger}=b_{e, f}^{\dagger} a_{f, h}^{\dagger}$;

(g) $\left(a_{f, h}^{\dagger} a b b_{e, f}^{\dagger}\right)_{f, f}^{\dagger}=b b_{e, f}^{\dagger} a_{f, h}^{\dagger} a$.

Proof. This proof follows from Corollary 3.1 in the same way as in the proof of Theorem 2.6.

Notice that in Corollary 3.6 the condition $(a b)_{e, h}^{\dagger}=b_{e, f}^{\dagger}\left(a_{f, h}^{\dagger} a b b_{e, f}^{\dagger}\right)_{f, f}^{\dagger} a_{f, h}^{\dagger}$ can be replaced with some equivalent conditions from Corollary 3.5.

Arghiriade [1] proved that $(a b)^{\dagger}=b^{\dagger} a^{\dagger}$ holds if and only if $a^{*} a b b^{*}$ is EP, i.e., $a^{*} a b b^{*}$ commutes with its Moore-Penrose inverse. The conjecture is: Is there a similarly result involving weighted MP inverse?

4. Conclusions. In this paper, we consider a number of necessary and sufficient conditions related to the reverse order law for the Moore-Penrose inverse in $C^{*}$-algebras. Applying this result we obtain the equivalent conditions for the reverse order rule for the weighted Moore-Penrose inverse of elements in $C^{*}$-algebras. All of these results are already known for complex matrices and some of them for closed range linear bounded operators on Hilbert spaces. However, we used the different technique in proving the results. In the theory of complex matrices various authors used the matrix rank to prove the equivalent conditions related to the reverse order law. In the case of linear bounded operators on Hilbert spaces, it seems that the method of operator matrices is very useful. In this paper, we applied a purely algebraic technique. It could be interesting to extend this work to the Moore-Penrose 
inverse and the weighted Moore-Penrose inverse of a triple product.

Acknowledgement. We are grateful to the referee for helpful comments concerning the paper.

\section{REFERENCES}

[1] E. Arghiriade. Remarques sur l'inverse généralisée d'un produit de matrices. Atti Accad. Naz. Lincei Rend. Cl. Sci. Fis. Mat. Natur. Ser VIII, 42:621-625, 1967.

[2] R.H. Bouldin. The pseudo-inverse of a product. SIAM J. Appl. Math., 25:489-495, 1973.

[3] R.H. Bouldin. Generalized inverses and factorizations. Recent Applications of Generalized Inverses, Pitman Ser. Res. Notes in Math., 66:233-248, 1982.

[4] D.S. Djordjević. Further results on the reverse order law for generalized inverses. SIAM J. Matrix Anal. Appl., 29(4):1242-1246, 2007.

[5] D.S. Djordjević and N.Č. Dinčić. Reverse order law for the Moore-Penrose inverse. J. Math. Anal. Appl., 361(1):252-261, 2010.

[6] D.S. Djordjević and V. Rakočević. Lectures on generalized inverses. Faculty of Sciences and Mathematics, University of Niš, 2008.

[7] T.N.E. Greville. Note on the generalized inverse of a matrix product. SIAM Rev., 8:518-521, 1966.

[8] R.E. Harte and M. Mbekhta. On generalized inverses in C*-algebras. Studia Math., 103:71-77, 1992.

[9] R.E. Hartwig. Block generalized inverses. Arch. Rational Mech. Anal., 61:197-251, 1976.

[10] S. Izumino. The product of operators with closed range and an extension of the reverse order law. Tohoku Math. J., 34:43-52, 1982.

[11] J.J. Koliha, D.S. Djordjević, and D. Cvetković. Moore-Penrose inverse in rings with involution. Linear Algebra Appl., 426:371-381, 2007.

[12] D. Mosić and D.S. Djordjević. Moore-Penrose-invertible normal and Hermitian elements in rings. Linear Algebra Appl., 431(5-7):732-745, 2009.

[13] R. Penrose. A generalized inverse for matrices. Proc. Cambridge Philos. Soc., 51:406-413, 1955.

[14] W. Sun and Y. Wei. Inverse order rule for weighted generalized inverse. SIAM J. Matrix Anal. Appl., 19:772-775, 1998.

[15] Y. Tian. On mixed-type reverse-order laws for Moore-Penrose inverse of a matrix product. Int. J. Math. Math. Sci., 58:3103-3116, 2004.

[16] Y. Tian. Using rank formulas to characterize equalities for Moore-Penrose inverses of matrix product. Appl. Math. Comput., 147:581-600, 2004. 